\title{
Interfacial structures of acidic and basic aqueous solutions
}

\author{
Chuanshan $\operatorname{Tian}^{1}$, $\mathrm{Na} \mathrm{Ji}^{1}$, Glenn Waychunas ${ }^{2}$, and \\ Y. Ron Shen ${ }^{1}$. \\ ${ }^{1}$ Department of Physics, University of California, \\ Berkeley, CA 94720 \\ ${ }^{2}$ Geochemistry Department, Lawrence Berkeley National Laboratory, \\ Berkeley, CA 94720
}

\begin{abstract}
Phase-sensitive sum-frequency vibrational spectroscopy was used to study water/vapor interfaces of $\mathrm{HCl}, \mathrm{HI}$, and $\mathrm{NaOH}$ solutions. The measured imaginary part of the surface spectral responses provided direct characterization of $\mathrm{OH}$ stretch vibrations and information about net polar orientations of water species contributing to different regions of the spectrum. We found clear evidence that hydronium ions prefer to emerge at interfaces. Their $\mathrm{OH}$ stretches contribute to the "ice-like" band in the spectrum. Their charges create a positive surface field that tends to reorient water molecules more loosely bonded to the topmost water layer with oxygen toward the interface, and thus enhances significantly the "liquid-like" band in the spectrum. Iodine ions in solution also like to appear at the interface and alter the positive surface field by forming a
\end{abstract}


narrow double-charge layer with hydronium ions. In $\mathrm{NaOH}$ solution, the observed weak change of the "liquid-like" band and disappearance of the "ice-like" band in the spectrum indicates that $\mathrm{OH}$ - ions must also have excess at the interface. How they are incorporated in the interfacial water structure is however not clear.

\section{Introduction}

Water/vapor interfaces of acidic and basic aqueous solutions play a key role in environmental chemistry and atmospheric science. ${ }^{1,2}$ They have been the topics of extensive theoretical and experimental studies in recent years, ${ }^{3-8}$ but good understanding of the water interfacial structure at the molecular level is still lacking. The most concerned question is whether $\mathrm{H}^{+}$and $\mathrm{OH}^{-}$ions would emerge at the interface and affect the interfacial hydrogen-bonding network of water. Molecular dynamics (MD) simulations predicted that protons $\left(\mathrm{H}^{+}\right)$could appear at the interface in the form of hydronium $\left(\mathrm{H}_{3} \mathrm{O}^{+}\right)$, but $\mathrm{OH}^{-}$should be repelled from the water surface. $^{3,9-12}$ The details, such as the depth profile of ion concentrations, depend very much on the molecular model and interaction potentials assumed. ${ }^{9-12}$ Experiments carried out with scanning tunneling microscopy $(\mathrm{STM}){ }^{13}$, X-ray spectroscopy ${ }^{14,15}$, and attenuated total reflection (ATR) ${ }^{8}$ 
have been used to verify the theoretical prediction. They however suffer from either the water surface being too dynamic or the techniques not being sufficiently surface-specific.

Sum-frequency vibrational spectroscopy (SFVS) has been proven to be a highly surface-specific and versatile probe for liquid interfaces, and is the only technique available to acquire surface vibrational spectra of liquids. ${ }^{5,6,16}$ Using SFVS, several research groups have successfully obtained vibrational spectra of water/vapor interfaces of acid, basic and salt solutions. ${ }^{5-8,17,18}$ In all cases, the SFVS spectrum appears to roughly consist of three spectral features in the $\mathrm{OH}$ stretch region: a relatively sharp peak at $3700 \mathrm{~cm}^{-1}$ attributed to the dangling $\mathrm{OH}$ protruding at the surface, and two broad bands at $\sim 3200$ and $\sim 3450 \mathrm{~cm}^{-1}$ from bonded $\mathrm{OH}$ often labeled as ice-like and liquid-like bands, respectively. ${ }^{16}$ When the acid concentration in water was sufficiently high ( $\mathrm{pH} \leq 2$ with $\mathrm{HCl}$ ), strong enhancement of both ice-like and liquid-like bands were observed..$^{7,8,11,18}$ Appearance of hydronium ions at the surface was used to explain the enhancement. ${ }^{7,8,11}$ In basic solution, only the liquid-like band displayed slight changes when $\mathrm{pH}$ is sufficiently high $(\mathrm{pH} \geq 13) .^{7}$ It was believed that at high $\mathrm{pH}, \mathrm{OH}^{-}$anions might have emerged at the interface with enough concentration to significantly distort the interfacial hydrogen-bonding network of water. ${ }^{7}$ Salt solutions have also been investigated. Results showed that ions of small size like to stay in the 
bulk, while negative ions with large radius, such as $\mathrm{Br}^{-}$and $\mathrm{I}^{-}$prefer to stay close to the surface. ${ }^{6}$

How the water interfacial structure would change upon emergence of various types of ions at the water/vapor interface is however still not clear. Interpretation of the SF spectrum for the same interface often varies from group to group. The problem arises because the conventional SFVS yields only an intensity spectrum, proportional to the absolute square of the surface nonlinear susceptibility, $\left|\chi_{S}^{(2)}\left(\omega_{I R}\right)\right|^{2}$, with $\chi_{S}^{(2)}\left(\omega_{I R}\right)$ being complex. To deduce information on resonances from $\left|\chi_{S}^{(2)}\left(\omega_{I R}\right)\right|^{2}$, one usually assumes discrete resonances and fits the spectrum. ${ }^{6-8,19}$ Unfortunately, such fitting is often not unique. The reason is that $\mathrm{OH}$ stretch resonances of a water interface are not discrete. They form a broad continuum because of the existence of widely varying geometry and strength of hydrogen bonds connecting interfacial water molecules to their neighbors. Properly characterizing the continuum resonances from fitting of $\left|\chi_{S}^{(2)}\left(\omega_{I R}\right)\right|^{2}$ is generally not possible. One could approximate the continuum resonances by discrete resonances, but without specifying $a$ priori the resonance frequencies and the signs of their resonant amplitudes, the fitting would not be unique. Thus when different groups fit the same SF intensity spectrum for a water interface with discrete resonances, they often obtained different results, and accordingly, 
different interpretations. ${ }^{7,8}$ Obviously the situation calls for a direct measurement of $\operatorname{Im} \chi_{S}^{(2)}\left(\omega_{I R}\right)$, which playing the same role as the imaginary part of the dielectric constant for absorption or emission spectroscopy, contains the desired resonant information.

We have recently developed a phase-sensitive (PS) SFVS technique that allows us to measure spectra of both the amplitude and the phase of $\chi_{S}^{(2)}\left(\omega_{I R}\right)$, and hence the spectra of $\operatorname{Re} \chi_{S}^{(2)}\left(\omega_{I R}\right)$ and $\operatorname{Im} \chi_{S}^{(2)}\left(\omega_{I R}\right){ }^{20,21} \mathrm{We}$ have applied it to the neat water/vapor interface and obtained more detailed understanding on the structure of the interface. ${ }^{22}$ We can now use the neat water/vapor interface as a reference in our SFVS study of water/vapor interfaces of acid and base solutions. From the spectral changes resulting from dissolving acid or base into water, we can learn how various types of ions appearing at the interface can perturb the interfacial water structure. We shall focus on the $\operatorname{Im} \chi_{S}^{(2)}\left(\omega_{I R}\right)$ spectra of $\mathrm{HCl}, \mathrm{NaOH}$, and $\mathrm{HI}$ solutions in which protons, hydroxyls and iodine ions like to come to the interface.

\section{Background of Phase-Sensitive Sum-Frequency Vibrational Spectroscopy}

Consider two input beams overlapping at a surface, one at a fixed visible frequency and the other tunable over vibrational resonances in the 
infrared. The SF output is given by $I\left(\omega_{S F}\right) \propto\left|\chi_{\text {eff }}^{(2)}\right|^{2}$, with ${ }^{23}$

$\chi_{\text {eff }}^{(2)}=\left[\vec{L}\left(\omega_{S F}\right) \cdot \hat{e}\left(\omega_{S F}\right)\right] \cdot \vec{\chi}_{S}^{(2)}:\left[\hat{e}\left(\omega_{v i s}\right) \cdot \vec{L}\left(\omega_{v i s}\right)\right]\left[\hat{e}\left(\omega_{I R}\right) \cdot \vec{L}\left(\omega_{I R}\right)\right]$

where $\vec{\chi}_{S}^{(2)}$ is the surface nonlinear susceptibility, and $\vec{L}(\omega)$ and $\hat{e}(\omega)$ are the transmission Fresnel factor and the polarization unit vector of the beam at $\omega$, respectively. With $\omega_{I R}$ near resonances, we can write

$$
\vec{\chi}_{S}^{(2)}=\vec{\chi}_{N R}^{(2)}+\sum_{q} \frac{\vec{A}_{q}}{\omega_{I R}-\omega_{q}+i \Gamma_{q}}
$$

if the resonances are discrete, and

$$
\vec{\chi}_{S}^{(2)}=\vec{\chi}_{N R}^{(2)}+\int \frac{\vec{A}_{q} \rho\left(\omega_{q}\right)}{\omega_{I R}-\omega_{q}+i \Gamma_{q}} d \omega_{q}
$$

if the resonances form a continuum. Here, $\ddot{\chi}_{N R}^{(2)}$ describes the nonresonant contribution. $\vec{A}_{q}, \omega_{q}$, and $\Gamma_{q}$ represent the amplitude, frequency, and damping constant of the $q^{\text {th }}$ vibrational resonance, respectively, and $\rho\left(\omega_{q}\right)$ is the density of modes at $\omega_{q}$.

In general, it is difficult to properly deduce characteristics of the resonances from fitting a $\left|\ddot{\chi}_{s}^{(2)}\right|^{2}$ spectrum using Eq.(2) or (3) if the resonances strongly overlap or form a continuum. For characterization of the resonances, one would like to know $\operatorname{Im} \ddot{\chi}_{s}^{(2)}$, instead of $\left|\vec{\chi}_{s}^{(2)}\right|^{2}$. This requires knowledge of both the amplitude, $\left|\vec{\chi}_{S}^{(2)}\right|$, and the phase, $\Phi_{S}$, of $\vec{\chi}_{s}^{(2)}$, both of which should be obtained directly from measurement. It is clear that $\Phi_{s}$ can be deduced from interference of the SF signal from the sample with that from a reference. 
Depicted in Fig. 1 is a possible scheme for the phase measurement of $\ddot{\chi}_{s}^{(2)} \cdot{ }^{21}$ It involves insertion of a reference crystal and a phase plate in the incoming beam path of a SFVS setup using the collinear beam geometry. The SF output generated from both the reference and the sample is given by

$$
\begin{aligned}
& S\left(\omega_{I R}\right) \propto\left|\chi_{S}^{(2)}(\omega)+C e^{i \Phi} \chi_{\operatorname{Re} f}^{(2)}(\omega)\right|^{2} \\
& \quad=\left|\chi_{S}^{(2)}\left(\omega_{I R}\right)\right|^{2}+\left|C \chi_{\operatorname{Re} f}^{(2)}\left(\omega_{I R}\right)\right|^{2}+2\left|C \chi_{S}^{(2)} \chi_{\operatorname{Re} f}^{(2)}\right| \cos \left[\Phi_{S}\left(\omega_{I R}\right)-\Phi_{\operatorname{Re} f}\left(\omega_{I R}\right)-\Phi\left(\omega_{I R}\right)\right]
\end{aligned}
$$

where $\chi_{S}^{(2)}=\left|\chi_{S}^{(2)}\right| e^{i \Phi_{S}}, \chi_{\mathrm{Re} f}^{(2)}=\left|\chi_{\mathrm{Re} f}^{(2)}\right| e^{i \Phi_{\mathrm{Re} f}}, \mathrm{C}$ is a constant depending on the beam profiles and $\Phi$ is a relative phase that can be adjusted by the phase plate. Because $\left|\chi_{S}^{(2)}\left(\omega_{I R}\right)+C e^{i \Phi} \chi_{\operatorname{Re} f}^{(2)}\left(\omega_{I R}\right)\right|^{2},\left|\chi_{S}^{(2)}\left(\omega_{I R}\right)\right|^{2}$, and $\left|C \chi_{\operatorname{Re} f}^{(2)}\left(\omega_{I R}\right)\right|^{2}$ can be measured separately, and $\Phi_{\operatorname{Re} f}\left(\omega_{I R}\right)$ and $\Phi\left(\omega_{I R}\right)$ are known, $\Phi_{S}\left(\omega_{I R}\right)$ can be readily deduced. The spectra of $\operatorname{Re} \tilde{\chi}_{S}^{(2)}\left(\omega_{I R}\right)$ and $\operatorname{Im} \ddot{\chi}_{S}^{(2)}\left(\omega_{I R}\right)$ can then be obtained.

The details of our PS-SFVS setup are described in Ref. 21. As seen in Fig. 1, a green beam $(532 \mathrm{~nm})$ and a tunable IR beam $(2.5-3.6 \mu \mathrm{m})$ derived from a picosecond Nd:YAG laser/optical parametric amplifier system propagated collinearly through a y-cut quartz plate and then onto the sample. The SF signal generated from the y-cut quartz, serving as a reference, interfered with that from the sample in the reflected direction. 
The interference signal was detected by a photomultiplier/gated integrator system after spectral filtering. A fused silica phase plate inserted between the sample and the y-cut quartz was used to vary $\Phi$. The SF output was normalized against that from a z-cut quartz plate.

The nonvanishing elements of $\vec{\chi}_{s}^{(2)}$ for water/vapor interfaces are $\chi_{S, Y Y Z}^{(2)}=\chi_{S, X X Z}^{(2)}, \chi_{S, Y Z Y}^{(2)}=\chi_{S, X Z X}^{(2)}, \chi_{S, Z Y Y}^{(2)}=\chi_{S, Z X X}^{(2)}$, and $\chi_{S, Z Z Z}^{(2)}$.

where $\mathrm{Z}$ is along the surface normal. We shall focus on $\chi_{S, Y Y Z}^{(2)}$ which can be measured by the SSP polarization combination (denoting S-, S-, and P-polarized SF output, visible input, and IR input, respectively). We note that the SF signal is proportional to $\left|\chi_{\text {eff }}^{(2)}\right|^{2}$, and $\chi_{\text {eff }}^{(2)}$ in Eq.(1) is related to $\chi_{s}^{(2)}$ through the Fresnel factors that depend on the beam geometry. In order to avoid confusion and be able to compare our spectra with those reported in the literature, we shall present our spectra with the Fresnel factors removed, i.e., we shall focus our discussion on $\chi_{s}^{(2)}$ instead of $\chi_{\text {eff }}^{(2)}$.

Aqueous solutions were held in a glass cell. Beams interrogating the water/vapor interface went through holes in the top cover of the cell. In preparing the samples, the cell was soaked in strong acid $\left(98 \% \mathrm{H}_{2} \mathrm{SO}_{4}+\right.$ NoChromix) for more than two days, then rinsed thoroughly with distilled water $(18.3 \mathrm{M} \Omega \cdot \mathrm{cm})$, and finally blow-dried with nitrogen gas. 
With such treatment, the sample surfaces were found free of detectable contaminants. Because $\mathrm{HI}$ in water is sensitive to room light, we prepared and kept the HI solution always in dark.

\section{Results}

We present in Figs. 2-4 the measured spectra of $\left|\chi_{S}^{(2)}\right|^{2}, \operatorname{Re} \chi_{S}^{(2)}$, and $\operatorname{Im} \chi_{s}^{(2)}$, for water/vapor interfaces of aqueous solutions of $1.2 \mathrm{M} \mathrm{HCl}, \mathrm{HI}$, and $\mathrm{NaOH}$, respectively. For comparison, we also plotted in each figure the spectra for the neat water/vapor interface. In all cases, our $\left|\chi_{S}^{(2)}\right|^{2}$ spectra, which have the Fresnel coefficients removed, agree well with those obtained by others. ${ }^{7,8,11}$ The spectra of all cases appear qualitatively similar, all having an ice-like and a liquid-like feature peaked at $\sim 3200$ and $\sim 3400 \mathrm{~cm}^{-1}$. Comparison with the neat water case shows that both features are significantly enhanced for $\mathrm{HCl}$ and $\mathrm{HI}$ solutions and only weakly altered for $\mathrm{NaOH}$ solution. In contrast, the corresponding $\operatorname{Im} \chi_{s}^{(2)}$ spectra exhibit little changes above $3500 \mathrm{~cm}^{-1}$ in all cases, but significant changes below $3450 \mathrm{~cm}^{-1}$ for $\mathrm{HCl}$ and $\mathrm{HI}$ solutions, including a stronger negative liquid-like band for $\mathrm{HCl}$ and $\mathrm{HI}$ solutions, and a flip of sign in the ice-like region. Comparison of the $\operatorname{Im} \chi_{S}^{(2)}$ spectra for $\mathrm{HCl}$ and $\mathrm{HI}$ solutions shows a stronger negative strength for $\mathrm{HCl}$ in the $\sim 3200-3500 \mathrm{~cm}^{-1}$ region and a stronger negative strength for $\mathrm{HI}$ in the $\sim 3100-3200 \mathrm{~cm}^{-1}$ region. For the $\mathrm{NaOH}$ solution, the $\operatorname{Im} \chi_{s}^{(2)}$ spectrum is 
somewhat less negative between 3200 and $3500 \mathrm{~cm}^{-1}$ compared to the neat water case and shows a negative, but nearly vanishing, ice-like band below $3200 \mathrm{~cm}^{-1}$.

\section{Discussion}

For water/vapor interfaces of $\mathrm{HCl}, \mathrm{HI}$, and $\mathrm{NaOH}$ solutions, the spectral changes observed in Figs. 2-4 with respect to the neat water case must come from ions appearing at the interfaces. It is believed that $\mathrm{H}^{+}, \mathrm{I}^{-}$, and perhaps also $\mathrm{OH}^{-}$can preferentially reside at a water/vapor interface. They could disturb the interfacial hydrogen $(\mathrm{H})$-bonding structure of water either by their physical presence or by the surface field they create. $^{7,8,12,18,24}$

In the following discussion, we shall use the neat water/vapor interface always as a reference. To facilitate the discussion, therefore, we need to briefly review the spectra and structure of the neat water/vapor interface deduced from our earlier PS-SFVS measurement. ${ }^{22}$ We have found that while the $\left|\chi_{s}^{(2)}\right|^{2}$ spectrum for neat water/vapor interface (displayed in Figs. 2a, 3a, and $4 \mathrm{a}$ as reference) is the same as those reported by others, the $\operatorname{Im} \chi_{s}^{(2)}$ spectrum (in Figs. $2 c, 3 c$, and $4 c$ ) is very different from those of others obtained from fitting of the $\left|\chi_{S}^{(2)}\right|^{2}$ spectrum. ${ }^{7,8}$ The $\operatorname{Im} \chi_{s}^{(2)}$ spectrum allows us to construct a qualitative picture of the interfacial structure: The water surface appears as a highly 
distorted ice surface. The topmost layer is covered with DAA and DDA molecules. (Here, D and A denote donor and acceptor H-bonds, respectively, with which water molecules connect to the neighbors.) The subsequent layer has DDAA molecules symmetrically or asymmetrically donor-bonded or singly donor-bonded to molecules in the topmost layer. These molecules are largely responsible for the observed SF spectra because further down into the liquid, molecular ordering at the surface rapidly decays away and approaches that of the isotropic bulk. In the $\operatorname{Im} \chi_{s}^{(2)}$ spectrum, the sharp peak at $3700 \mathrm{~cm}^{-1}$ can be identified as the $\mathrm{OH}$ stretch of the dangling $\mathrm{OH}$ at the surface, the $3450-3700 \mathrm{~cm}^{-1}$ region mainly as donor-bonded $\mathrm{OH}$ stretches of DDA and DAA molecules, the $3200-3450 \mathrm{~cm}^{-1}$ region mainly as asymmetrically D-bonded DDAA molecules, and 3000-3200 $\mathrm{cm}^{-1}$ regions mainly as symmetrically donor-bonded DDAA molecules. We emphasize here that the overall spectrum results from a continuous band of $\mathrm{OH}$ stretch resonances of interfacial water molecules that are $\mathrm{H}$-bonded to neighbors with a wide variety of different geometries and strengths. Therefore, the above description is only a crude approximation. Molecules ascribed here to the specific spectral regions also contribute to some extent to the neighboring spectral regions. For example, both symmetrically and asymmetrically bonded DDAA molecules should contribute to the $\operatorname{Im} \chi_{s}^{(2)}$ spectrum around $3200 \mathrm{~cm}^{-1}$, one positively and the other negatively so that the 
combined $\operatorname{Im} \chi_{S}^{(2)}$ is nearly zero.

We now compare the spectra of water/vapor interfaces of aqueous solutions with those of neat water. The spectral change then allows us to learn how ions emerging at the interfaces perturb the interfacial structure. We consider first the $\mathrm{HCl}$ acid solution. It was found earlier that there was no detectable structural change of the water/vapor interface with addition of up to $1.7 \mathrm{M} \mathrm{NaCl}$ in water. ${ }^{25}$ This indicates that both $\mathrm{Na}^{+}$and $\mathrm{Cl}^{-}$are largely repelled from the interface. In $\mathrm{HCl}$ solution, however, protons are likely to have excess at the interface as suggested by molecular dynamics simulations. ${ }^{9-11}$ They can be readily incorporated into the interfacial H-bonding network as hydrated ions in the form of $\mathrm{H}_{3} \mathrm{O}^{+}$ (Eigen form, hydronium) and/or $\mathrm{H}_{5} \mathrm{O}_{2}{ }^{+}$(Zundel form, $\left.\mathrm{H}_{2} \mathrm{O}-\mathrm{H}-\mathrm{OH}_{2}{ }^{+}\right){ }^{7,8,11,18}$ These hydrated ions can influence the interfacial spectra directly through their own $\mathrm{OH}$ stretches and less directly through reorientation of interfacial water molecules by the surface field they create. Recent SFVS studies ${ }^{7}$ showed enhancement of both ice-like and liquid-like bands in the $\left|\chi_{S}^{(2)}\right|^{2}$ spectrum due to solvation of $\mathrm{HCl}$ in water, which was an indication that $\mathrm{H}^{+}$ions had emerged at the interface to modify the water interfacial structure. Shultz and coworkers understood the enhancement as due to reorientation of interfacial water molecules by the $\mathrm{H}^{+}$-created surface field. ${ }^{18}$ Richmond and coworkers decomposed the 
$\left|\chi_{S}^{(2)}\right|^{2}$ spectrum into discrete resonances, and attributed the enhancement of the 3200 and $3330 \mathrm{~cm}^{-1}$ modes to effects of the strong surface field and strong electrostatic interactions between hydrated protons $\left(\mathrm{H}_{3} \mathrm{O}^{+}\right.$and $\mathrm{H}_{5} \mathrm{O}_{2}{ }^{+}$) and water molecules at the interface. ${ }^{7}$ Allen and coworkers fitted the spectrum with a different set of discrete resonances and suggested that the enhancement at $3200 \mathrm{~cm}^{-1}$ came from the surface field effect as well as contributions from $\mathrm{OH}$ stretch vibrations of $\mathrm{H}_{3} \mathrm{O}^{+}$and $\mathrm{H}_{5} \mathrm{O}_{2}^{+}$at the interface. ${ }^{8,11}$ Ishiyama and Morita recently reported their MD simulations on the water/vapor interface of $\mathrm{HCl}$ solution. ${ }^{12}$ Their calculated $\left|\chi_{S}^{(2)}\right|^{2}$ spectrum agree qualitatively with the experimental one, and the calculated $\operatorname{Im} \chi_{S}^{(2)}$ spectra also resemble that deduced from decomposition of the $\left|\chi_{s}^{(2)}\right|^{2}$ spectrum by Richmond's group. ${ }^{7} \quad$ (See Fig.5, where we compare the $\operatorname{Im} \chi_{s}^{(2)}$ spectra obtained by different groups. The signs of the resonant amplitudes were not specified in Ref. 8. In composing the $\operatorname{Im} \chi_{S}^{(2)}$ spectrum from the information given in Ref. 8, we assume that all the bonded $\mathrm{OH}$ modes have negative amplitude as we have measured.)

Our $\left|\chi_{s}^{(2)}\right|^{2}$ spectrum in Fig. 2a for the water/vapor interface of a $1.2 \mathrm{M}$ $\mathrm{HCl}$ solution is essentially the same as that obtained by other groups, but the measured $\operatorname{Im} \chi_{S}^{(2)}$ spectrum is different from those of others deduced from the $\left|\chi_{s}^{(2)}\right|^{2}$ spectrum assuming discrete resonances, ${ }^{7,8}$ and also from 
that of MD simulation ${ }^{12}$, as shown in Fig. 5. (We note that significant differences, including the difference in the signs of the resonant amplitudes, between our directly measured $\operatorname{Im} \chi_{s}^{(2)}$ spectrum and those of others are also evident for the neat water/vapor interface.) In comparison with the spectrum of the neat water/vapor interface also displayed in Fig. 2c, the dangling $\mathrm{OH}$ peak at $\sim 3700 \mathrm{~cm}^{-1}$ hardly changes, but the broad band due to bonded $\mathrm{OH}$ stretches in the $\left|\chi_{S}^{(2)}\right|^{2}$ spectrum is significantly enhanced. From the spectra of $\operatorname{Re} \chi_{s}^{(2)}$ and $\operatorname{Im} \chi_{s}^{(2)}$, it is obvious that the enhancement in the higher frequency region $\left(\geq 3200 \mathrm{~cm}^{-1}\right)$ comes from enhancement of $\operatorname{Im} \chi_{s}^{(2)}$ and enhancement in the lower frequency region $\left(\leq 3250 \mathrm{~cm}^{-1}\right)$ comes from $\operatorname{Re} \chi_{s}^{(2)}$.

The $\operatorname{Im} \chi_{s}^{(2)}$ spectra are more informative because it directly reflects the resonance profile as described in Eq.(3). Comparing the $\operatorname{Im} \chi_{s}^{(2)}$ spectra of $\mathrm{HCl}$ solution and neat water, we find (1) there is hardly any change above $3500 \mathrm{~cm}^{-1}$ except for a slight increase of the shoulder around 3600 $\mathrm{cm}^{-1}$, (2) the negative liquid-like band between 3200 and $23450 \mathrm{~cm}^{-1}$ becomes significantly stronger for the $\mathrm{HCl}$ solution, and (3) the ice-like band below $3200 \mathrm{~cm}^{-1}$ flips from positive to negative with $\mathrm{HCl}$ in water and terminates at $\sim 3100 \mathrm{~cm}^{-1}$.

Using the $\operatorname{Im} \chi_{s}^{(2)}$ spectrum in relation to the structure of neat water/vapor interface as references, we can deduce information on how 
the interfacial structure of the $\mathrm{HCl}$ solution differs from that of neat water from the spectral changes. As mentioned earlier, $\mathrm{H}^{+}$likes to emerge at the interface. Knowing that the spectrum above $3500 \mathrm{~cm}^{-1}$ comes mainly from DAA and DDA water molecules in the topmost layer at the surface, its insignificant change indicates that the surface concentration of $\mathrm{H}^{+}$or hydrated $\mathrm{H}^{+}$is not high enough to physically perturb the structure of the topmost layer. This is supported by the result of MD simulation that the excess proton concentration at the surface should be less than $4 \%$ of a monolayer. ${ }^{12}$ The surface field created by interfacial $\mathrm{H}^{+}$ions cannot affect DAA and DDA molecules in the topmost layer, but can reorient the more loosely H-bonded DDAA molecules in the adjacent layer with $\mathrm{O} \rightarrow \mathrm{H}$ toward the bulk liquid, thus enhancing the negative liquid-like band between 3200 and $3500 \mathrm{~cm}^{-1}$. It is less likely to be able to reorient DDAA molecules contributing to the ice-like band because of their stronger H-bonding to neighbors. ${ }^{26}$ The observed flip of the ice-like band below $3200 \mathrm{~cm}^{-1}$ from positive to negative could be due to contribution of $\mathrm{OH}$ stretches of hydronium $\left(\mathrm{H}_{3} \mathrm{O}^{+}\right)$ions appearing at the interface. They have preferred orientation with $\mathrm{O} \rightarrow \mathrm{H}$ pointing into the liquid and therefore contribute negatively to the respective spectral region. In recent years, there has been a great deal of discussion on the existence of hydronium ions and their $\mathrm{OH}$ stretch frequencies. Infrared spectroscopic studies on clusters $(\mathrm{n}=5)^{27}$ and bulk $\mathrm{HCl}$ solutions ${ }^{28}$ suggest the presence of 
hydronium ions with a stretch frequency around $\sim 2900 \mathrm{~cm}^{-1}$. Raman studies found a very broad band (called proton continuum) below $\sim 3200$ $\mathrm{cm}^{-1}$ which was attributed to $\mathrm{H}_{3} \mathrm{O}^{+}$ad $\mathrm{H}_{5} \mathrm{O}_{2}{ }^{+8,29}$ The spectral change we observe in the ice-like band region suggests that the surface hydronium ions do exist and should have their $\mathrm{OH}$ stretch frequencies in the range of $3000-3250 \mathrm{~cm}^{-1}$. It is possible that hydronium ions at the interface with no acceptor bond and weaker donor bonds to neighbors would have higher $\mathrm{OH}$ stretch frequencies than in the bulk.

To check the validity of the above assignment, we tried to single out contribution from hydronium to the spectrum by removing the surface field effect. This can be done by comparing the spectrum of $1.2 \mathrm{M} \mathrm{HCl}$ solution with a linear combination of spectra for $\mathrm{HCl}$ and $\mathrm{NaI}$ solutions. From previous SFVS studies of NaI solution, ${ }^{22}$ it is known that $\mathrm{I}^{-}$ions prefer to come to the surface and create a negative surface field. The spectral change with respect to the neat water interface mainly comes from the surface field reorienting the interfacial DDAA water molecules. ${ }^{22}$ On the other hand, in $\mathrm{HCl}$ solution, the surface field created by excess $\mathrm{H}^{+}$is positive and induces a spectral change opposite to that in NaI solution. Thus if we choose a proper linear combination of the two spectra for $\mathrm{HCl}$ and $\mathrm{NaI}$ solutions, we can expect cancellation of the surface field effect in the combined spectrum. Then, in comparing the 
combined spectrum with that of neat water, the observed difference would have to come from $\mathrm{OH}$ stretches of the hydronium ions. In Fig. 6a, we show separately the $\operatorname{Im} \chi_{s}^{(2)}$ spectra for $1.2 \mathrm{M} \mathrm{HCl}$ and $2.1 \mathrm{M} \mathrm{NaI}$ solutions. The two are obviously different in the $3000-3600 \mathrm{~cm}^{-1}$ region. We then present in Fig. $6 \mathrm{~b}$ the combined $\operatorname{Im} \chi_{s}^{(2)}$ spectrum composed of the average of the two spectra in Fig. $6 \mathrm{a}$ for $1.2 \mathrm{M} \mathrm{HCl}$ and $2.1 \mathrm{M} \mathrm{NaI}$ solutions and compare it with that of neat water. The two spectra in Fig. 6b differ only below $3250 \mathrm{~cm}^{-1}$ and around $3600 \mathrm{~cm}^{-1}$; the surface field effect that affects mainly the spectral region $3200-3500 \mathrm{~cm}^{-1}$ has indeed been suppressed. The difference spectrum of the two, also shown in Fig. 6 b, displays a negative band below $3250 \mathrm{~cm}^{-1}$ that can be attributed to $\mathrm{OH}$ stretches of interfacial hydronium ions and a small positive bump presumably arising from disturbance of interfacial DAA water molecules by the hydronium ions. ${ }^{30}$ Figure $6 \mathrm{c}$ shows a cartoon describing the water/vapor interfacial structure of $\mathrm{HCl}$ solution.

We next consider the water/vapor interface of the HI acid solution. Both $\mathrm{H}^{+}$and $\mathrm{I}^{-}$would have excess at the surface. However, $\mathrm{H}^{+}$must have more surface excess because as we described earlier, it takes $2.1 \mathrm{M} \mathrm{NaI}$ in water to produce enough surface excess $\mathrm{I}^{-}$ions to create the same field strength as that of surface excess $\mathrm{H}^{+}$ions produced by $1.2 \mathrm{M} \mathrm{HCl}$. For an HI solution with equal bulk concentrations of $\mathrm{H}^{+}$and $\mathrm{I}^{-}$, more $\mathrm{H}^{+}$than $\mathrm{I}^{-}$ 
must appear at the water/vapor interface. This is supported by MD simulation. ${ }^{12}$ The surface ions form a double-charge layer with a positive surface field that could reorient DDAA molecules with $\mathrm{O} \rightarrow \mathrm{H}$ pointing towards the liquid bulk and enhance the negative liquid-like band in the $\operatorname{Im} \chi_{S}^{(2)}$ spectrum.

Our $\left|\chi_{S}^{(2)}\right|^{2}$ spectrum for the 1.2M HI solution in Fig. 3a is the same as those obtained by others, but our $\operatorname{Im} \chi_{s}^{(2)}$ spectrum is again different from those of others deduced from fitting of the $\left|\chi_{S}^{(2)}\right|^{2}$ spectrum. ${ }^{8}$ Compared to that of neat water, the $\operatorname{Im} \chi_{S}^{(2)}$ spectrum of the HI solution shows little change above $3500 \mathrm{~cm}^{-1}$ except for the shoulder at $\sim 3600 \mathrm{~cm}^{-1}$, but an enhanced negative strength in the $3200-3500 \mathrm{~cm}^{-1}$ liquid-like region. Below $3200 \mathrm{~cm}^{-1}$, the ice-like band becomes negative. The result supports the picture that more $\mathrm{H}^{+}$than $\mathrm{I}^{-}$ appear at the surface, producing a positive surface field that changes the liquid-like spectral feature through reorientation of the asymmetrically bonded DDAA water molecules. This picture differs from the one predicted by MD simulation of Dang et $\mathrm{al}^{4}$ that shows a negative surface field formed by $\mathrm{H}^{+}$than $\mathrm{I}^{-}$, but agrees with the MD simulations of Morita et al ${ }^{12}$. The hydronium ions formed by $\mathrm{H}^{+}$at the interface again make the ice-like band appear negative and the positive shoulder at $3600 \mathrm{~cm}^{-1}$ stronger.

Compared to the $\operatorname{Im} \chi_{s}^{(2)}$ spectrum of the $1.2 \mathrm{M} \mathrm{HCl}$ solution (see 
Fig.7a), the enhancement in the liquid-like region is somewhat less, but the negative ice-like band is stronger and so is the positive shoulder at $\sim 3600 \mathrm{~cm}^{-1}$. This is an indication that there are more hydronium ions at the interface of the $\mathrm{HI}$ solution than the $\mathrm{HCl}$ solution, but the surface field is weaker. We can understand the observation as follows. Due to Coulomb interaction, the presence of $\mathrm{H}^{+}$(or hydronium ions) at the interface helps attract $\mathrm{I}^{-}$ions to the interface and vice versa. ${ }^{31}$ Therefore, for the same molar concentration of $\mathrm{HI}, \mathrm{HCl}$, and $\mathrm{NaI}$ in solutions, more $\mathrm{H}^{+}$and $\mathrm{I}^{-}$ions are expected at the interface of the $\mathrm{HI}$ solution than $\mathrm{H}^{+}$and $\mathrm{I}^{-}$ions at the interfaces of the $\mathrm{HCl}$ and $\mathrm{NaI}$ solutions, respectively. Saykally and coworkers using second harmonic generation to monitor the amount of $\mathrm{I}^{-}$ions at the vapor/water interfaces of $\mathrm{NaI}$ and $\mathrm{HI}$ solutions, and indeed found more $\mathrm{I}^{-}$ions in the HI case. ${ }^{31}$. However, since both $\mathrm{H}^{+}$ and $\mathrm{I}^{-}$preferentially appear at the surface, they are likely to be close to each other, forming a relatively thin double-charge layer. Compared to the $\mathrm{HCl}$ case, even though the surface concentration of $\mathrm{H}^{+}$is higher, the surface field effect could still be weaker, as actually seen from the weaker liquid-like band in the $\operatorname{Im} \chi_{S}^{(2)}$ spectra. MD simulations support the above description. ${ }^{11,12}$ Morita et al shows that the HI solution has a larger surface excess density of $\mathrm{H}^{+}\left(\rho / \rho_{\text {bulk }}=2.3\right)$ than the $\mathrm{HCl}$ solution $\left(\rho / \rho_{\text {bulk }}=1.55\right)$, and the average gap between $\mathrm{H}^{+}$and $\mathrm{I}^{-}$is only 1.5 $\AA$, much smaller than that between $\mathrm{H}^{+}$and $\mathrm{Cl}^{-}(4.5 \AA)$. Thus the surface 
potential is lower at the interface of the HI solution. We present in Fig. $7 \mathrm{~b}$ a cartoon describing the interfacial water structure of the HI solution.

Finally, we consider the water/vapor interface of $1.2 \mathrm{M} \mathrm{NaOH}$ solution. This is a case more difficult to understand. Even in the bulk, how $\mathrm{OH}^{-}$is incorporated in an hydrogen-bonding structure of water is not very clear ${ }^{32}$. Traditionally, $\mathrm{OH}^{-}$is thought of being a "mirror image" of $\mathrm{H}^{+}$in water, capable of hydrogen-bonding to four neighboring water molecules by three acceptor bonds and one donor bond. However, this picture has been challenged by recent theoretical and experimental works, which proposed that $\mathrm{OH}^{-}$could have one donor and four acceptor bonds ${ }^{32,33}$. The bonding structure of $\mathrm{OH}^{-}$at the water/vapor interface is even less clear. MD simulation predicts that $\mathrm{OH}^{-}$should be repelled from the interface ${ }^{11}$. SFVS studies on $1.3 \mathrm{M} \mathrm{NaOH}$ solution, on the other hand, found clear reduction of the liquid-like band in the $\left|\chi_{s}^{(2)}\right|^{2}$ spectrum $^{7,11}$, indicating that $\mathrm{OH}^{-}$had emerged at the interface. The reduction was attributed to $\mathrm{OH}^{-}$ inducing random orientation of water molecules at the interface ${ }^{7}$.

$\operatorname{Our}\left|\chi_{s}^{(2)}\right|^{2}$ spectrum of an $1.2 \mathrm{M} \mathrm{NaOH}$ in Fig. 4 is the same as those of others seeing a reduction of the liquid-like band, but the $\operatorname{Im} \chi_{s}^{(2)}$ spectrum in comparison with the neat water case shows more clearly a change in the disappearance of the ice-like band below $3200 \mathrm{~cm}^{-1}$, in addition to the weak reduction of the negative liquid-like band. The latter can be understood to be due to the surface field effect created by excess $\mathrm{OH}^{-}$at 
the interface that tends to reorient interfacial water molecules with $\mathrm{O} \rightarrow \mathrm{H}$ toward the interface. This rather small change, in comparison with those observed in $\mathrm{NaI}, \mathrm{HCl}$, and $\mathrm{HI}$ solutions, indicates that the field resulting from surface excess of $\mathrm{OH}^{-}$is much less than in the other cases, but it is still opposite to the prediction of MD simulation that there should be a surface depletion of $\mathrm{OH}^{-}$. The disappearance of the ice-like band must also be due to appearance of $\mathrm{OH}^{-}$at the interface. It is not likely that the weak surface field can reorient the symmetrically bonded DDAA water molecules responsible for the ice-like band, and is presumably the result of how $\mathrm{OH}^{-}$is incorporated into the hydrogen-bonding structure of water at the interface. Randomization of interfacial structure by five-coordinated $\mathrm{OH}^{-}$probably is probably not the reason because changes in other spectral regions are very weak. Since DDAA molecules symmetrically donor-bonded to the topmost DDA and DAA molecules mainly contribute to the ice-like band in the neat water case, we suspect that in $\mathrm{NaOH}$ solution, $\mathrm{OH}^{-}$ions may have come to the interface to replace such DDAA molecules and thus reduce the ice-like band. It is also possible that incorporation of $\mathrm{OH}^{-}$ions in the interfacial hydrogen-bonding structure may affect the $\mathrm{OH}$ stretches of water molecules bonded to them and contribute to the change of the ice-like band. However, these are pure conjectures. Theoretical help is clearly needed to understand the interfacial structure of $\mathrm{NaOH}$ solution. 


\section{Conclusions}

We used the PS-SFVS technique to study water/vapor interfaces of 1.2 $\mathrm{M} \mathrm{HCl}, \mathrm{HI}$ and $\mathrm{NaOH}$ solutions, and obtained in the $\mathrm{OH}$ stretch region not only the $\left|\chi_{S}^{(2)}\right|^{2}$ spectrum, but also the spectra of $\operatorname{Re} \chi_{S}^{(2)}$ and $\operatorname{Im} \chi_{S}^{(2)}$. The $\operatorname{Im} \chi_{s}^{(2)}$ spectrum directly reveals the resonance characteristics, and is therefore more informative. While our $\left|\chi_{s}^{(2)}\right|^{2}$ spectra are essentially identical to those obtained by others, our $\operatorname{Im} \chi_{s}^{(2)}$ spectra are different from those deduced from fitting of the $\left|\chi_{S}^{(2)}\right|^{2}$ spectra by others. Accordingly, our understanding of the water interfacial structures of these solutions is also different.

With the neat water/vapor interface taken as a reference, we found that protons in $\mathrm{HCl}$ solution like to appear in the form of hydronium ions at the interface and create a positive surface field, which tends to reorient the interfacial water molecules and alters the liquid-like band of the spectrum. The hydronium ions themselves oriented with $\mathrm{O} \rightarrow \mathrm{H}$ toward the liquid contribute to the negative ice-like band in the $\operatorname{Im} \chi_{s}^{(2)}$ spectrum and an enhanced shoulder below the dangling $\mathrm{OH}$ peak. In the case of $\mathrm{HI}$ solution, both $\mathrm{H}^{+}$and $\mathrm{I}^{-}$prefer to appear at the interface. They form a double-charge layer creating again a positive surface field that changes the liquid-like band in the spectrum. The hydronium ions at the 
interface also affect the spectrum the same way as in the $\mathrm{HCl}$ case. For the $\mathrm{NaOH}$ solution, the Im (2) $S \chi$ spectrum indicates preferential appearance of $\mathrm{OH}$ - at the interface, contrary to the prediction of MD simulation. How $\mathrm{OH}$ - gets incorporated in the hydrogen-bonding structure of the water interface and alters the spectrum is however difficult to visualize.

This work demonstrates how PS-SFVS is needed to study water interfaces. The experimentally determined $\operatorname{Im}(2) S \chi$ spectrum properly describes the extremely broad band of $\mathrm{OH}$ stretch resonances originating from continuously varying bonding geometry and strength interfacial water molecules connect with neighbors. It is more of a challenge to theoretical studies of water interfaces. Hopefully, theoretical reproduction of the spectrum could also provide information on how different interfacial water species contribute to different spectral regions. Results from this work will also be used as the basis and references for future studies of other water interfaces including water/liquid and water/solid interfaces.

\section{Acknowledgement}

This work was supported by NSF grant through WaterCAMPWS. GAW's work was partially supported by the U.S. Department of Energy under Contract No. DE-AC02$05 \mathrm{CH} 11231$. 


\section{Figures:}

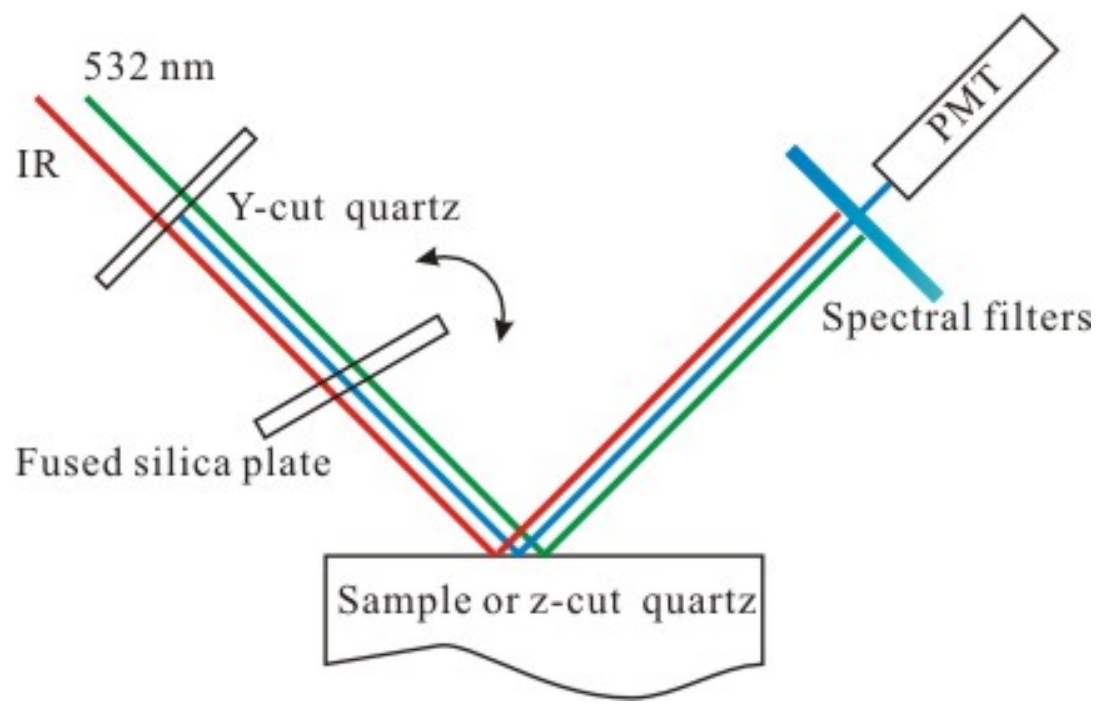

Figure 1. Schematic of the PS-SFVS setup. 


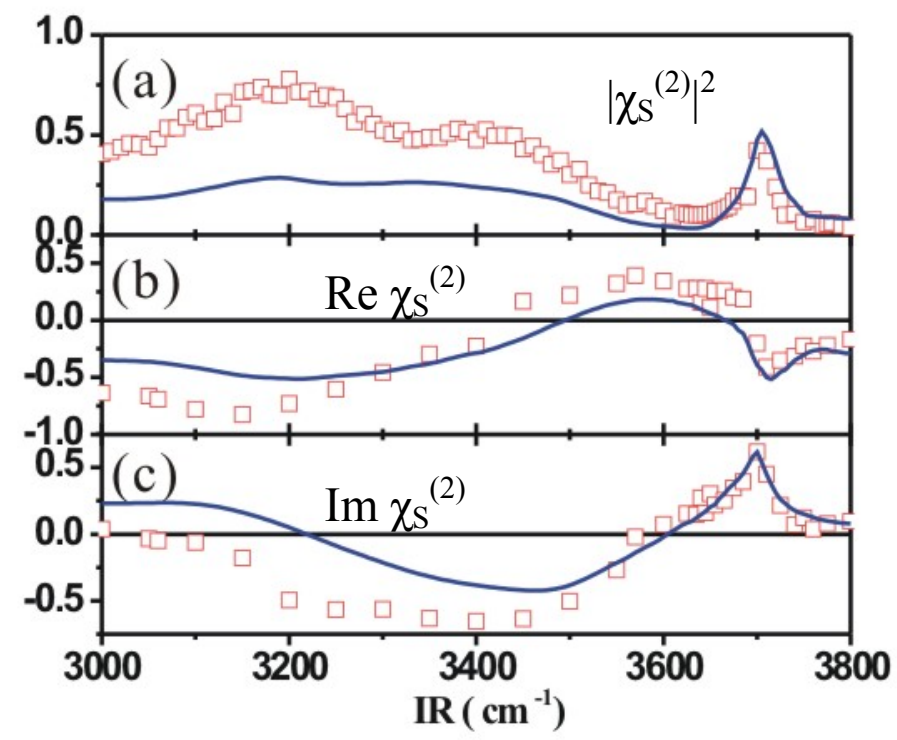

Figure 2. Spectra of $(\mathrm{a})\left|\chi_{s}^{(2)}\right|^{2},(\mathrm{~b}) \operatorname{Re} \chi_{s}^{(2)}$ and $(\mathrm{c}) \operatorname{Im} \chi_{s}^{(2)}$ for the water/vapor interfaces of 1.2 M HCl solution (symbols) and neat water (lines). 


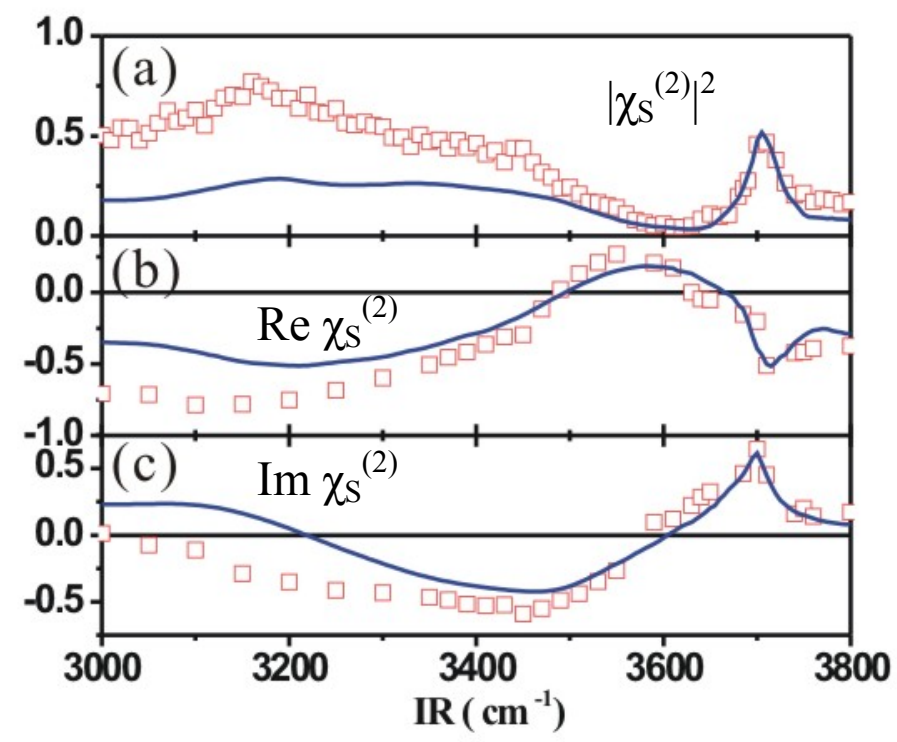

Figure 3. Spectra of (a) $\left|\chi_{s}^{(2)}\right|^{2}$, (b) $\operatorname{Re} \chi_{s}^{(2)}$ and (c) $\operatorname{Im} \chi_{s}^{(2)}$ for the water/vapor interfaces of 1.2 M HI solution (symbols) and neat water (lines). 


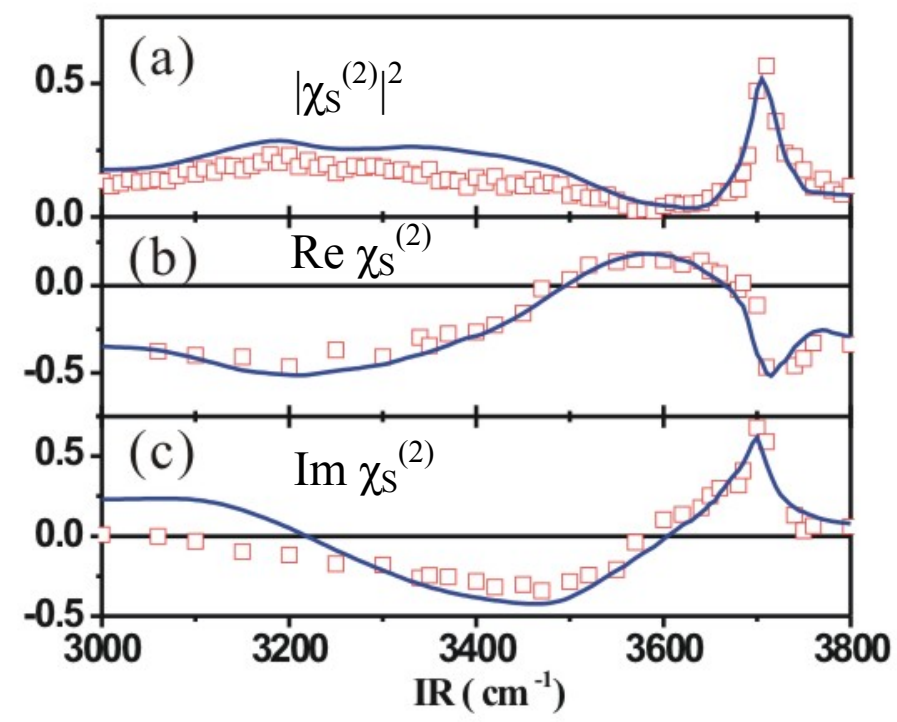

Figure 4. Spectra of $(\mathrm{a})\left|\chi_{s}^{(2)}\right|^{2},(\mathrm{~b}) \operatorname{Re} \chi_{s}^{(2)}$ and $(\mathrm{c}) \operatorname{Im} \chi_{s}^{(2)}$ for the water/vapor interfaces of $1.2 \mathrm{M} \mathrm{NaOH}$ solution (symbols) and neat water (lines). 

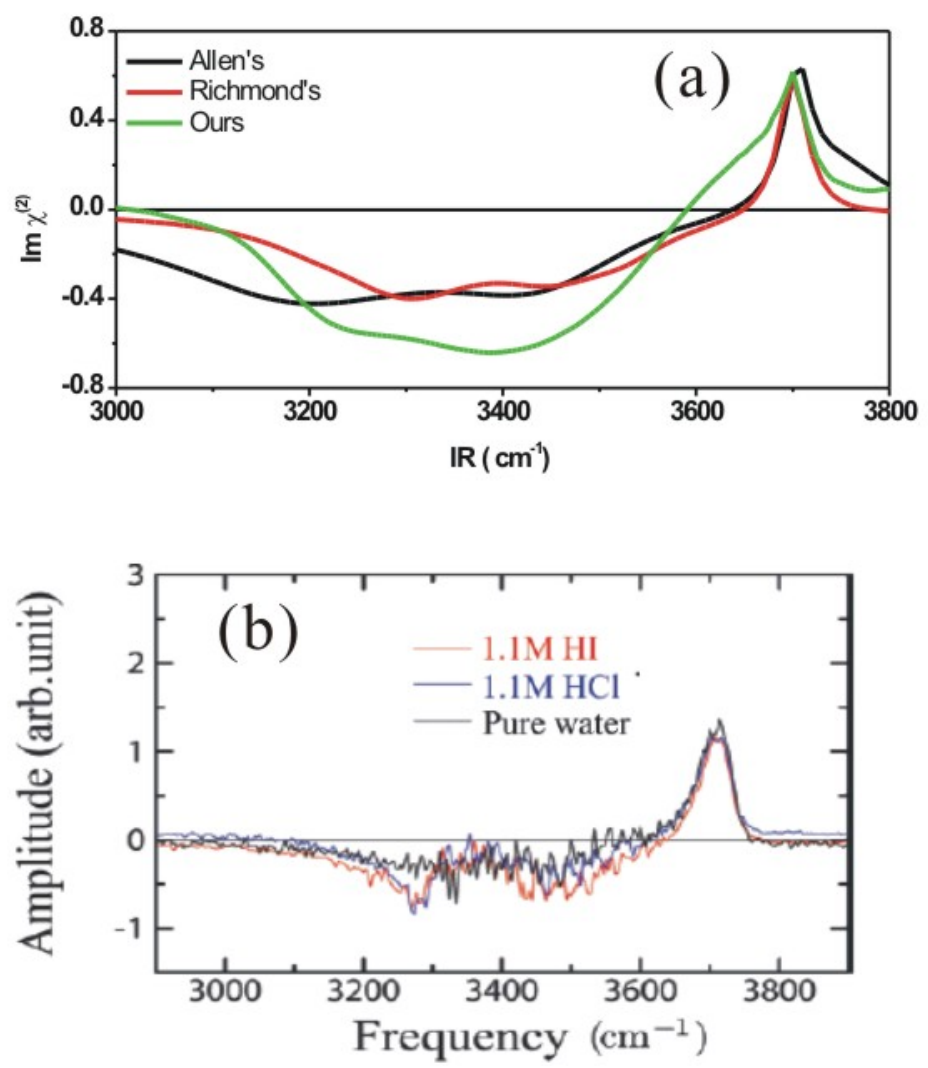

Figure 5. Comparison of $\operatorname{Im} \chi_{s}^{(2)}$ spectra of the water/vapor interface of 1.2 $\mathrm{M} \mathrm{HCl}$ solution obtained (or deduced from spectral information given) by different groups. MD simulation by Morita's group is shown in (b). 

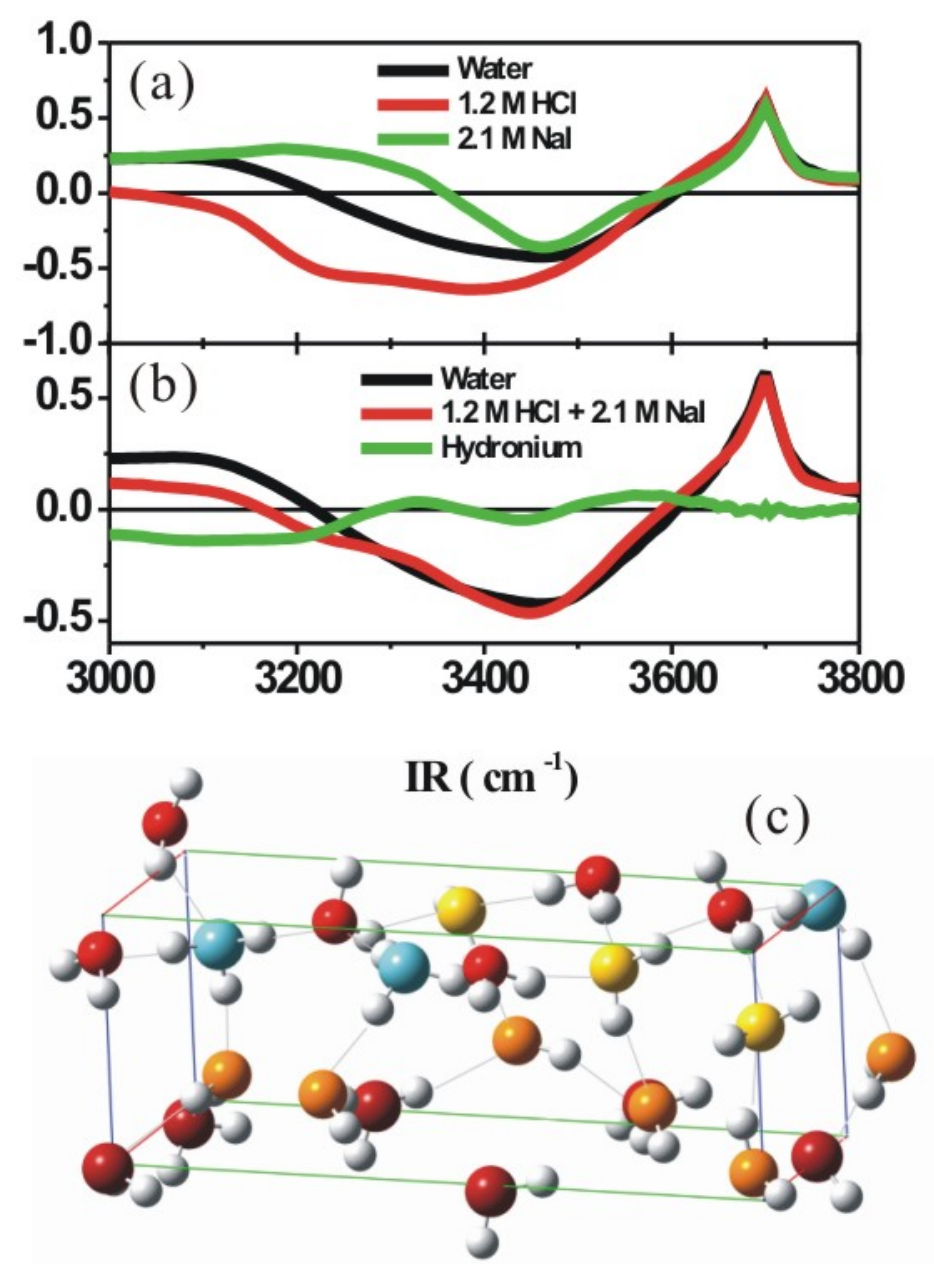

Figure 6. (a) $\operatorname{Im} \chi_{s}^{(2)}$ spectra of water/vapor interfaces of $1.2 \mathrm{M} \mathrm{HCl}$ solution (red line) and 2.1 M NaI solution (green line). (b) $\operatorname{Im} \chi_{s}^{(2)}$ spectrum of water/vapor interfaces of near water (black line), 1:1 linear superposition of $\operatorname{Im} \chi_{s}^{(2)}$ spectra for $1.2 \mathrm{M} \mathrm{HCl}$ and $2.1 \mathrm{M} \mathrm{NaI}$ solutions (red line) and the difference spectrum deduced from the above two (green line). (c) Cartoon depicting the water/vapor interfacial structure of the $\mathrm{HCl}$ solution. Water molecules in different layers are coded with different colors while oxygens of hydronium ions are painted blue. 


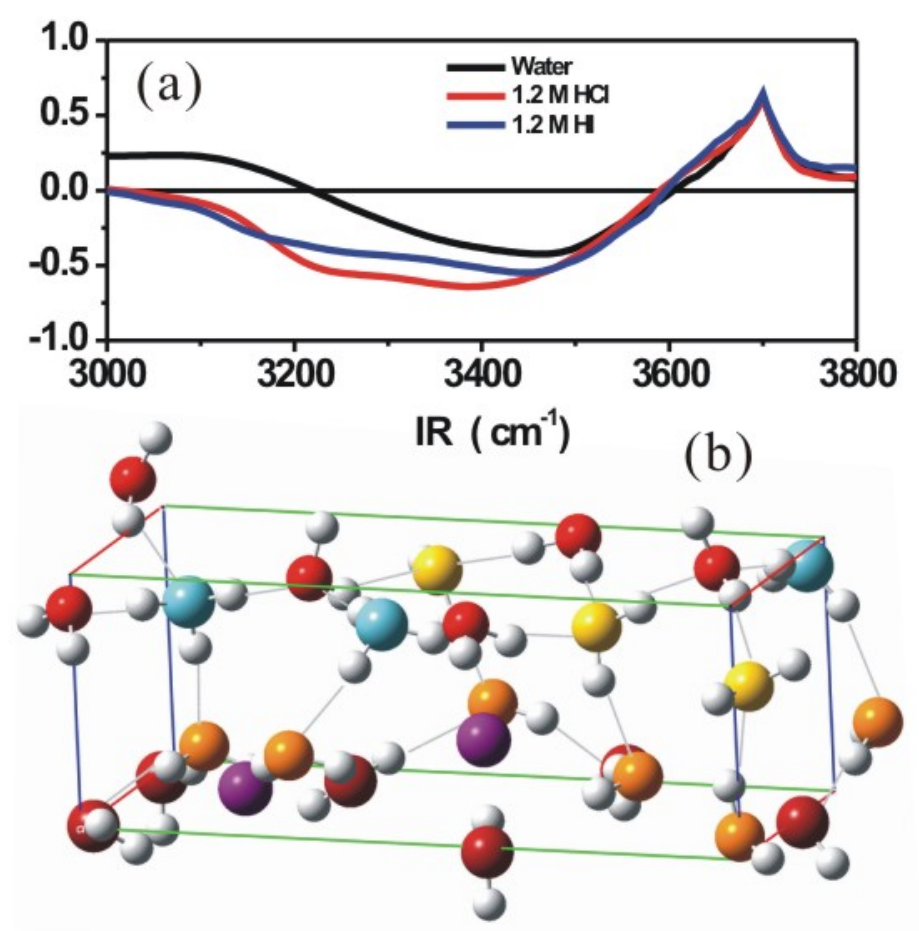

Figure 7. (a) Comparison of $\operatorname{Im} \chi_{s}^{(2)}$ spectra of water/vapor interfaces of 1.2 $\mathrm{M} \mathrm{HCl}$ (red line) and $\mathrm{HI}$ (blue line) solutions. (b) Cartoon depicting the water/vapor interfacial structure of the HI solution. Water molecules in different layers are coded with different colors while oxygens of hydronium ions are painted blue and iodine ions painted purple. 


\section{References:}

(1) Irwin, J. G.; Williams, M. L. Environ Pollut 1988, 50, 29-59.

(2) The Chemistry of Acid Rain: Sources and Atmospheric Processes; American Chemical

Society: Washington, DC, 1987.

(3) Jungwirth, P.; Tobias, D. J. Chemical Reviews 2006, 106, 1259-1281.

(4) Chang, T. M.; Dang, L. X. Chemical Reviews 2006, 106, 1305-1322.

(5) Shen, Y. R.; Ostroverkhov, V. Chemical Reviews 2006, 106, 1140-1154.

(6) Gopalakrishnan, S.; Liu, D. F.; Allen, H. C.; Kuo, M.; Shultz, M. J. Chemical Reviews 2006, 106, 1155-1175.

(7) Tarbuck, T. L.; Ota, S. T.; Richmond, G. L. Journal of the American Chemical Society 2006, 128, 14519-14527.

(8) Levering, L. M.; Sierra-Hernandez, M. R.; Allen, H. C. Journal of Physical Chemistry C 2007, 111, 8814-8826.

(9) Dang, L. X. Journal of Chemical Physics 2003, 119, 6351-6353.

(10) Petersen, M. K.; Iyengar, S. S.; Day, T. J. F.; Voth, G. A. Journal of Physical Chemistry B 2004, 108, 14804-14806.

(11) Mucha, M.; Frigato, T.; Levering, L. M.; Allen, H. C.; Tobias, D. J.; Dang, L. X.; Jungwirth, P. Journal of Physical Chemistry B 2005, 109, 7617-7623.

(12) Ishiyama, T.; Morita, A. Journal of Physical Chemistry A 2007, 111, 9277-9285.

(13) Allongue, P. Phys Rev Lett 1996, 77, 1986-1989.

(14) Cappa, C. D.; Smith, J. D.; Messer, B. M.; Cohen, R. C.; Saykally, R. J. Journal of Physical Chemistry B 2006, 110, 1166-1171.

(15) Cappa, C. D.; Smith, J. D.; Messer, B. M.; Cohen, R. C.; Saykally, R. J. Journal of Physical Chemistry A 2007, 111, 4776-4785.

(16) Du, Q.; Superfine, R.; Freysz, E.; Shen, Y. R. Phys Rev Lett 1993, 70, 2313-2316.

(17) Baldelli, S.; Schnitzer, C.; Shultz, M. J.; Campbell, D. J. Journal of Physical Chemistry B 1997, 101, 10435-10441.

(18) Baldelli, S.; Schnitzer, C.; Shultz, M. J. Chemical Physics Letters 1999, 302, 157-163.

(19) Wei, X.; Miranda, P. B.; Shen, Y. R. Phys Rev Lett 2001, 86, 1554-1557.

(20) Ostroverkhov, V.; Waychunas, G. A.; Shen, Y. R. Phys Rev Lett 2005, 94, -.

(21) Ji, N.; Ostroverkhov, V.; Chen, C. Y.; Shen, Y. R. Journal of the American Chemical Society 2007, 129, 10056-+.

(22) Ji, N.; Ostroverkhov, V.; Tian, C. S.; Shen, Y. R. Phys Rev Lett 2008.

(23) Shen, Y. R. In Frontier in Laser Spectroscopy; Inguscio, T. W. H. a. M., Ed.: North Holland, Amsterdam, 1994, pp 139-165.

(24) Liu, D. F.; Ma, G.; Levering, L. M.; Allen, H. C. Journal of Physical Chemistry B 2004, 108, 2252-2260.

(25) Raymond, E. A.; Richmond, G. L. Journal of Physical Chemistry B 2004, 108, 5051-5059.

(26) In a previous study on a $2.1 \mathrm{M} \mathrm{NaI}$ solution [Ref. 22], it was found that the surface field created by I- ions emerging at the surface was not strong enough to alter the spectrum below $3200 \mathrm{~cm}^{-1}$ significantly. The surface field created by protons at the surface in a $1.2 \mathrm{M} \mathrm{HCl}$ solution is not expected to be stronger.

(27) Headrick, J. M.; Diken, E. G.; Walters, R. S.; Hammer, N. I.; Christie, R. A.; Cui, J.; 
Myshakin, E. M.; Duncan, M. A.; Johnson, M. A.; Jordan, K. D. Science 2005, 308, 1765-1769.

(28) Kim, J.; Schmitt, U. W.; Gruetzmacher, J. A.; Voth, G. A.; Scherer, N. E. Journal of Chemical Physics 2002, 116, 737-746.

(29) Kanno, H. J Raman Spectrosc 1993, 24, 689-693.

(30) The slight increase around $3600 \mathrm{~cm}^{-1}$ could result from DAA molecules with a weakened donor bond due to the presence of hydrated protons in the neighborhood.

(31) Petersen, P. B.; Saykally, R. J. Journal of Physical Chemistry B 2005, 109, 7976-7980.

(32) Tuckerman, M. E.; Chandra, A.; Marx, D. Accounts Chem Res 2006, 39, 151-158.

(33) Botti, A.; Bruni, F.; Imberti, S.; Ricci, M. A.; Soper, A. K. J Mol Liq 2005, 117, 81-84. 